PREPARED FOR THE U.S. DEPARTMENT OF ENERGY, UNDER CONTRACT DE-AC02-76CH03073

PPPL-3891

PPPL-3891

UC-70

3D Adaptive Mesh Refinement Simulations

of Pellet Injection in Tokamaks

by

R. Samtaney, S.C. Jardin, P. Colella, and D.F. Martin

October 2003

NM|

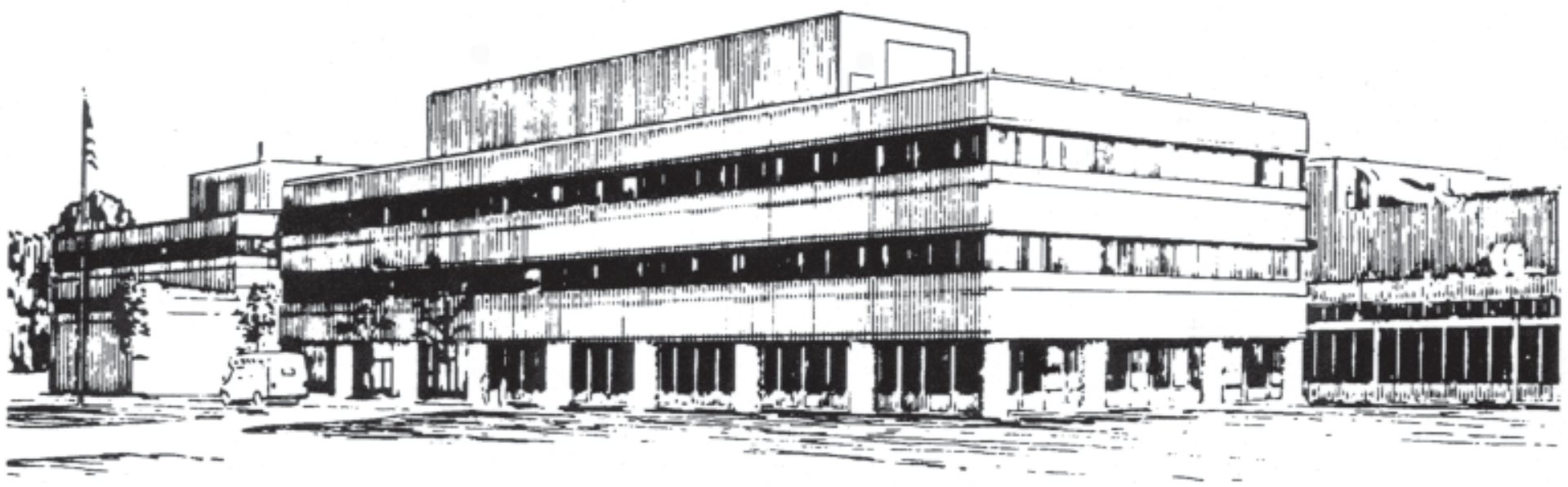

PRINCETON PLASMA PHYSICS LABORATORY PRINCETON UNIVERSITY, PRINCETON, NEW JERSEY 


\section{PPPL Reports Disclaimer}

This report was prepared as an account of work sponsored by an agency of the United States Government. Neither the United States Government nor any agency thereof, nor any of their employees, makes any warranty, express or implied, or assumes any legal liability or responsibility for the accuracy, completeness, or usefulness of any information, apparatus, product, or process disclosed, or represents that its use would not infringe privately owned rights. Reference herein to any specific commercial product, process, or service by trade name, trademark, manufacturer, or otherwise, does not necessarily constitute or imply its endorsement, recommendation, or favoring by the United States Government or any agency thereof. The views and opinions of authors expressed herein do not necessarily state or reflect those of the United States Government or any agency thereof.

\section{Availability}

This report is posted on the U.S. Department of Energy's Princeton Plasma Physics Laboratory Publications and Reports web site in Fiscal Year 2004. The home page for PPPL Reports and Publications is: http://www.pppl.gov/pub_report/

DOE and DOE Contractors can obtain copies of this report from:

U.S. Department of Energy

Office of Scientific and Technical Information

DOE Technical Information Services (DTIS)

P.O. Box 62

Oak Ridge, TN 37831

Telephone: (865) 576-8401

Fax: (865) 576-5728

Email: reports@adonis.osti.gov

This report is available to the general public from:

National Technical Information Service

U.S. Department of Commerce

5285 Port Royal Road

Springfield, VA 22161

Telephone: $1-800-553-6847$ or

(703) $605-6000$

Fax: (703) 321-8547

Internet: http://www.ntis.gov/ordering.htm 


\title{
3D Adaptive Mesh Refinement Simulations of Pellet Injection in Tokamaks
}

\author{
R. Samtaney ${ }^{\mathrm{a}}$ S. C. Jardin ${ }^{\mathrm{a}}$ P. Colella ${ }^{\mathrm{b}}$ D. F. Martin ${ }^{\mathrm{b}}$ \\ ${ }^{a}$ Princeton Plasma Physics Laboratory, Princeton, NJ 08543 \\ ${ }^{\mathrm{b}}$ Lawrence Berkeley National Laboratory, Berkeley, CA 94720
}

\begin{abstract}
We present results of Adaptive Mesh Refinement (AMR) simulations of the pellet injection process, a proven method of refueling tokamaks. AMR is a computationally efficient way to provide the resolution required to simulate realistic pellet sizes relative to device dimensions. The mathematical model comprises of single-fluid MHD equations with source terms in the continuity equation along with a pellet ablation rate model. The numerical method developed is an explicit unsplit upwinding treatment of the 8-wave formulation, coupled with a MAC projection method to enforce the solenoidal property of the magnetic field. The Chombo framework is used for AMR. The role of the $E \times B$ drift in mass redistribution during inside and outside pellet injections is emphasized.
\end{abstract}

Key words: Adaptive Mesh Refinement, MHD, Pellet Injection.

PACS: $52.30 . \mathrm{Cv}, 52.65 . \mathrm{Kj}$

\section{Introduction}

Injecting small pellets of frozen hydrogen into a tokamak is a proven method of fueling. Experimentally, it is known that the density distribution, after the pellet ablates upon encountering the high temperatures in a tokamak, is not consistent with the distribution inferred from assuming that the ablated material remains on the flux surfaces where the ablation occurred. The subsequent redistribution of mass is believed to be due to anomalous MHD processes. The mass redistribution is observed to be a sensitive function of the angle (with respect to the mid-plane) in which the pellet is injected $[1,2]$. It is this phenomenon which we seek to explain.

A previous three-dimensional computational investigation of pellet injection was performed by Strauss and Park [3]. They investigated the evolution of a

To appear in Computer Physics Communications, Elsevier Publishers 
large density "blob" representing the ionized pellet ablation cloud. However, they did not treat a moving pellet source and their resolution was relatively coarse. Our approach is to perform detailed simulations of the pellet injection process and quantify the MHD processes responsible for mass redistribution. We employ Adaptive Mesh Refinement (AMR) in our simulations to provide the resolution required to simulate realistic pellet sizes relative to device dimensions (typical ratios are $O\left(10^{-3}\right)$ ). In section 2 , we describe the physical problem and the mathematical model along with the pellet ablation model and discussion of initial and boundary conditions. In section 3, we describe the numerical method. In section 4, we present results from AMR simulations emphasizing the differences between inside and outside injections.

\section{Description of Problem and Mathematical Model}

\subsection{Physical Problem}

The physical problem we are dealing with involves the injection of frozen fuel pellets into a tokamak. The physical processes are broadly distinguished into the following two stages. The first stage is the ablation of mass at the pellet surface due to the high temperature background plasma encountered by the pellet. The ablated pellet mass, which is a neutral gas, is rapidly heated by electrons and ionizes to form plasma. The second stage is the redistribution

of the ablated pellet material by free streaming along the magnetic field lines and by anomalous MHD processes which cause mass flow across field lines and flux surfaces. The pellet ablation phenomenon of the first stage is considered well-understood [4,5], and as such we use existing ablation models. The thrust of the work described here is an accurate and efficient simulation of the second phase.

\subsection{Mathematical Model}

Our mathematical model consists of single fluid MHD equations with source terms in the continuity equation to model the mass injected into the system by the pellet, and source (sink) terms in the energy equations to model electron heating and corresponding cooling on flux surfaces. The equations are written below.

$$
\frac{\partial U}{\partial t}+\frac{\partial F_{j}(U)}{\partial x_{j}}=\frac{\partial F_{v, j}(U)}{\partial x_{j}}+S_{T}(U)+S_{\nabla \cdot \mathbf{B}}(U)+S_{\text {pellet }}(U)
$$


where the solution vector $U \equiv U\left(x_{1}, x_{2}, x_{3}, t\right) \equiv U\left(R, z, R_{0} \phi, t\right)$ is $U=\left\{\rho, \rho u_{i}, B_{i}, e\right\}^{T}$, and the flux vector $F_{j}(U)$ is given by

$$
F_{j}(U)=\left\{\begin{array}{c}
\rho u_{j} \\
\rho u_{i} u_{j}+p_{t} \delta_{i j}-B_{i} B_{j}+B_{T} B_{3} \delta_{i j}-B_{i} B_{T} \delta_{3 j}-B_{j} B_{T} \delta_{i 3} \\
u_{j} B_{i}-u_{i} B_{j}+B_{T} \delta_{i 3} u_{j}-B_{T} \delta_{3 j} u_{i} \\
\left(e+p+\frac{1}{2} B_{k} B_{k}\right) u_{j}-B_{j}\left(B_{k} u_{k}\right)+B_{T} B_{3} u_{j}-\left(B_{k} u_{k}\right) B_{T} \delta_{3 j}
\end{array}\right\}(2)
$$

In the above equations, $R, z, \phi$, are the radial, axial and toroidal coordinates, $R 0$ is the major radius; $\rho$ is the density, $u_{i}$ is the velocity, $B_{i}$ is the magnetic field, $p$ and $p_{t}$ are the pressure and total pressure, respectively, and $e$ is the total energy per unit volume of the plasma. For numerical stability and robustness, we have subtracted out the equilibrium toroidal component of the initial equilibrium magnetic field, $B_{T}\left(x_{i}, 0\right) \equiv g_{0} / R$. These equations are closed by the perfect gas equation of state,

$$
e=\frac{p}{\gamma-1}+\frac{\rho}{2} u_{k} u_{k}+\frac{1}{2} B_{k} B_{k}
$$

which we note does not include the contribution $1 / 2 B_{T}^{2}$. The flux vector $F_{v, j}(U)$ corresponds to the diffusive resistivity/viscosity terms and is omitted in the interest of brevity. The toroidal geometry terms are modeled in the source terms as

$$
S_{T}(U)=-\frac{1}{R}\left(\begin{array}{c}
\rho u_{R} \\
\rho u_{R}^{2}-\rho u_{\phi}^{2}-B_{R}^{2}+B_{\phi}^{2}+2 B_{\phi} B_{T} \\
\rho u_{R} u_{Z}-B_{R} B_{z} \\
2 \rho u_{R} u_{\phi}-2 B_{R} B_{\phi}-2 B_{R} B_{T} \\
0 \\
u_{R} B_{z}-B_{R} u_{z} \\
0 \\
\left(e+p_{t}\right) u_{R}-(\mathbf{B} \cdot \mathbf{u}) B_{R}+B_{T} B_{\phi} u_{R}
\end{array}\right)+\left(\frac{1}{R_{0}}-\frac{1}{R}\right) \frac{\partial F_{\phi}}{\partial \phi} .
$$

For a large aspect ratio tokamak, $S_{T}(U)$ is small but it contains essential toroidal effects which cause the in-out asymmetry discussed in Section 4. The source terms $S_{\nabla \cdot \mathbf{B}}(U)$, written below,

$$
S_{\nabla \cdot \mathbf{B}}(U)=-\nabla \cdot \mathbf{B}\left(\left\{0, B_{R}, B_{\phi}, B_{z}, u_{R}, u_{z}, u_{\phi}, u_{z},(B \cdot u)\right\}^{T}\right)
$$


are included because we use the symmetrization procedure of Godunov [6] which leads to the 8-wave formulation. This formulation was also used by Powell et al. [7] in their AMR implementation of ideal MHD. Finally, the source terms $S_{\text {pellet }}=\left\{S_{n} / n_{0}, 0,0,0,0,0,0, S_{e} / n_{0}\right\}^{T}$, where $n_{0}$ is some reference number density, correspond to the mass source and energy source/sink terms, and are described next.

\subsection{Pellet Ablation Model}

In the present model, the pellet is described by a sphere of frozen molecular hydrogen of radius $r_{p}$. The trajectory $x_{p}\left(x_{i}, t\right)$ of the pellet is prescribed with a given initial location $x_{p 0} \equiv x_{p}\left(x_{i}, 0\right)$ and constant velocity $u_{p}$. The density source term arises from the ablation of the pellet and is written in terms of number density, ( i.e., atoms per unit volume per unit time) as

$$
S_{n}=\dot{N} \delta\left(x-x_{p}\right)
$$

where the delta function is approximated as a Gaussian distribution centered over the pellet with a characteristic size equal to $10 r_{p}$. The ablation rate of the pellet, originally derived by Parks and Turnbull [4] and modified for hydrogen pellets by Kuteev [8] is given below (in atoms/sec)

$$
\dot{N}=-4 \pi r_{p}^{2} \frac{d r_{p}}{d t} 2 n_{m}=1.12 \times 10^{16} n_{e}^{0.333} T_{e}^{1.64} r_{p}^{1.33} M_{i}^{-0.333}
$$

where $n_{e}$ is the background plasma density in $\mathrm{cm}^{-3}, T_{e}$ is the background plasma electron temperature in $\mathrm{eV}, M_{i}$ is the atomic mass number in atomic units and $n_{m}=2.63 \times 10^{22} / \mathrm{cm}^{3}$ is the molecular density of frozen hydrogen. A useful approximation which eliminates the electron timescale from the problem is to consider the electron heat flux as being instantaneous compared to the other processes being computed. The time-asymptotic effect of the large electron heat flux is to make the temperature uniform along field lines, i.e., $T \equiv T(\psi)$. Thus, for single fluid equations, the temperature $T(\psi)$ in the volume $V_{\psi}$ between flux surfaces $\psi$ and $\psi+d \psi$ will equilibrate as the density changes while still conserving energy in the volume $V_{\psi}$. This leads to the following energy source terms in the energy equation

$$
S_{e}=3\left(S_{n} T(\psi)+n \dot{T}(\psi)\right) .
$$

The first term in $S_{e}$ corresponds to the localized increase in energy due to the heating of the ablated pellet mass, while the second term corresponds to a global adiabatic cooling of the entire flux surface. In practice, we compute the contribution due to the second term by separately solving a 1D model for the pellet injection assuming only classical processes are present. We then use 
table lookup and interpolation to compute the term $\dot{T}(\psi)$ in our 3D AMR simulation.

\subsection{Initial and Boundary Conditions}

The initial condition is a static equilibrium state. The initial magnetic field is written in terms of two function $\psi(R, z)$ and $g(R, z)$, i.e.,

$$
B=\frac{1}{R}(\hat{\phi} \times \nabla \psi+g \hat{\phi})
$$

These functions satisfy the Grad-Shafranov equation,

$$
R \frac{\partial}{\partial R} \frac{1}{R} \frac{\partial \psi}{\partial R}+\frac{\partial^{2} \psi}{\partial z^{2}}+R^{2} \frac{d p}{d \psi}+g \frac{d g}{d \psi}=0
$$

where $p \equiv p(\psi)$ and $g \equiv g(\psi)$. For a torus with rectangular cross-section of radial extent $2 a$ and axial extent of $2 b=2 \kappa a$ we may write $\psi(R, z)=$ $f(R) \cos (\pi z / \kappa a)$. Further, with $g(R, z)=g_{0}=$ constant we get

$$
R \frac{d}{d R}\left(\frac{1}{R} \frac{d f}{d R}\right)+\left(\frac{R_{0} \pi}{a}\right)^{2}\left(\alpha R^{2}-\frac{1}{4 \kappa^{2}}\right) f=0
$$

which permits a Frobenius-type series solution. The value of $\alpha$ is determined by imposing the boundary conditions $\psi=0$. The pressure is written as $p=\bar{p}+$ $p_{0} \psi^{2}$ where $\bar{p}$ is a small background pressure to avoid zero ion-acoustic speeds and $p_{0}=\alpha \pi^{2} /\left(2 a^{2} R_{0}^{2}\right)$. The toroidal field function $g_{0}=R_{0} \alpha \pi^{2}|\psi|_{\max } q_{0} /(2 a b)$, where $q_{0}(\approx 1)$ is the on-axis safety factor. Boundary conditions imposed are perfectly conducting walls in the radial/axial directions and periodic in the toroidal direction. In our simulations we use $\kappa=1, a / R 0=\pi / 9$, for which $\alpha=0.481509$.

\section{Numerical Method}

In this section, we focus on the evaluation of the hyperbolic flux terms $\left(F_{j}(U)\right)$ in Eqn. (1). We use a finite volume technique wherein each variable is stored at the cell center. The numerical fluxes of conserved quantities are obtained at the cell faces using a combination of the 8-wave formulation [6] and unsplit upwinding $[9,10]$. We define a vector of "primitive" variables $W=\left\{\rho, u_{i}, B_{i}, p\right\}^{T}$. Given the conserved quantities and all the source terms, i.e., $U_{\boldsymbol{i}}^{n}, S_{\boldsymbol{i}}^{n}$, (in this notation, $\boldsymbol{i}$ is a 3 -tuple correponding to the three dimensions), we want to compute a second-order accurate estimate of the fluxes: $F_{i+\frac{1}{2} e^{d}}^{n+\frac{1}{2}}(d$ indicates 
the d-th direction, $0 \leq d<2$ ) The first step is to compute $W_{i}^{n}$ in each cell, followed by fitting a linear profile in each cell subject to slope limiting. We then extrapolate the primitive and conserved variables at the cell faces using the normal derivative terms and the source terms at the cell centers, as follows.

$$
W_{\boldsymbol{i}, \pm, d}=W_{\boldsymbol{i}}^{n}+\frac{1}{2}\left( \pm I-A_{\boldsymbol{i}}^{d} \frac{\Delta t}{h}\right) P_{ \pm} \Delta^{d} W_{\boldsymbol{i}}, \quad U_{\boldsymbol{i}, \pm, d}=U\left(W_{\boldsymbol{i}, \pm, d}\right)+\frac{\Delta t}{2} S_{\boldsymbol{i}}^{n}(12)
$$

where $A_{i}^{d}=\left(\nabla_{W} U \nabla_{W} F^{d}\right)\left(W_{i}\right)$ and $P_{ \pm}(W)=\sum_{ \pm \lambda_{k}>0}\left(l_{k} \cdot W\right) r_{k}$, and $\Delta^{d} W_{\boldsymbol{i}}$ is the undivided but limited slope. The eigenvalues, and left and right eigenvectors of $A_{i}^{d}$ are $\lambda_{k}, l_{k}$, and $r_{k}$, respectively with $k=1 \cdots 8$ in the eight-wave formulation (see Powell et al. [7] for the left and right eigenvectors). We compute corrections to $U_{\boldsymbol{i}, \pm, d}$ corresponding to one set of transverse derivatives appropriate to obtain $(1,1,1)$ diagonal coupling:

$$
U_{\boldsymbol{i}, \pm, d_{1}, d_{2}}=U_{\boldsymbol{i}, \pm, d_{1}}-\frac{\Delta t}{3 h}\left(F_{i+\frac{1}{2} e^{d_{2}}}^{1 \mathbf{D}}-F_{i-\frac{1}{2} e^{d_{2}}}^{1 \mathbf{D}}\right), \quad d_{1} \neq d_{2}, 0 \leq d_{1}, d_{2}<3
$$

where $F_{\boldsymbol{i} \pm \frac{1}{2} \boldsymbol{e}^{d}}^{1 \mathbf{D}}=R P\left(U_{\boldsymbol{i},+, d}, U_{\boldsymbol{i}+\boldsymbol{e}^{d,-, d}}\right)$. The notation $F=R P\left(U_{L}, U_{R}\right)$ implies that the flux $F$ is evaluated by solving a linearized Riemann problem using $U_{L}$ and $U_{R}$ as left and right states, respectively. We next compute final corrections to $U_{i, \pm, d}$ due to transverse derivatives:

$$
U_{\boldsymbol{i}, \pm, d}^{n+\frac{1}{2}}=U_{\boldsymbol{i}, \pm, d}-\frac{\Delta t}{2 h}\left(F_{i+\frac{1}{2} e^{d_{1}, d_{2}}}-F_{\boldsymbol{i}-\frac{1}{2} e^{d_{1}, d_{2}}}\right),
$$

where $F_{\boldsymbol{i}+\frac{1}{2} e^{d_{1}, d_{2}}}=R P\left(U_{\boldsymbol{i},+, d_{1}, d_{2}}, U_{\boldsymbol{i}+\boldsymbol{e}^{d_{1},+, d_{1}, d_{2}}}\right)$, and $0 \leq d<3 ; d_{1} \neq d_{2} \neq d$. At this stage, we solve another Riemann problem at the cell faces using $U_{i,+, d}^{n+\frac{1}{2}}$ and $U_{i+e^{d},-, d}^{n+\frac{1}{2}}$ as the left and right states, respectively. The magnetic field obtained from the solution to the Riemann problem at $n+\frac{1}{2}$ at the cell faces is not guaranteed to be divergence free. We enforce the solenoidal property of the magnetic field by a MAC projection, using $\mathbf{B}$ at the cell faces to obtain a cell-centered monopole charge density. A Poisson solver is used to find a scalar field satisfying $\nabla^{2} \chi=\nabla \cdot \mathbf{B}$ with Neumann boundary conditions in the radial/axial directions and periodic in the toroidal direction. The magnetic field at the cell faces is then corrected according to $B_{i+\frac{1}{2} e^{d}}^{n+\frac{1}{2}}=B_{i+\frac{1}{2} e^{d}}^{n+\frac{1}{2}}-\nabla \chi$. Finally the fluxes at cell faces are obtained as $F_{i+\frac{1}{2} e^{d}}^{n+\frac{1}{2}}=F\left(U_{i+\frac{1}{2} e^{d}}^{n+\frac{1}{2}}\right)$ and the conserved quantity at the cell centers are updated using these fluxes. The Poisson equation in the projection step above is cast in a residual-correction form and solved using a multi-grid technique on each level in the AMR hierarchy. 
The residual smoothing is a Gauss-Seidel relaxation procedure with red-black ordering. When meshes cannot be coarsened any further, the Poisson solve is taken to convergence using a bottom-smoother which is a biconjugate gradient solver. We implemented the above method into the Chombo framework and have developed a second-order adaptive parallel MHD code. Chombo is a collection of $\mathrm{C}++$ libraries for implementing block-structured AMR finite difference calculations [11]. Particular care is taken in implementing coarsefine interface interpolations of appropriate order to ensure second-order accuracy. Furthermore, conservation at coarse-fine interfaces is maintained by flux-refluxing. This leads to a non-zero cell-centered $\nabla \cdot \mathbf{B}$ in coarse cells which are adjacent to coarse-fine boundaries, which being a set of codimension one does not significantly affect the accuracy of the solution.

\section{Simulation Results}

In this section, we present preliminary results from early to intermediate stages of pellet injection. The results discussed here correspond to a midpoint toroidal field of $0.23 \mathrm{~T}, \beta \approx 0.1$, and a pellet of $1 \mathrm{~mm}$ radius moving radially with a velocity of $3200 \mathrm{~m} / \mathrm{s}$ in a tokamak with minor radius of $a=0.26 \mathrm{~m}$. Two cases are discussed: one in which the pellet is initialized on the high field side (HFS or the so-called inside launch case) and the other in which the pellet is injected from the low field side (LFS or the "outside" launch case). Because the temperature of the plasma is low near the edges of the tokamak, we initialize the pellet at some radial distance inside the tokamak. This is merely to save computational effort and have interesting dynamics take place relatively quickly. In both the LFS and the HFS case, the initial location of the pellet is on the same flux surface so that the pellet encounters the same initial temperature in both the LFS and HFS cases. Based on preliminary tests which suggested that the energy sink term provides only a small contribution, but is nonetheless computationally expensive to evaluate and occasionally leads to noisy solutions, we omitted the sink term in the results presented here.

Fig. 1 shows a density isosurface, viewed radially inwards, at times $t=2,20,60$ (time is normalized by the Alfvén time) for the HFS case. The outlines of the various meshes in the calculation are also shown in Fig. 1. At $t=2$ the pellet ablated mass is roughly in the shape of an ellipsoid with its major axis aligned along the magnetic field lines. The pellet cloud is a localized region of high $\beta$ with the dominant mass motion being along the magnetic field lines. As time progresses, the ablated mass moves parallel to the magnetic field at speeds of about one-third of the local acoustic speed. (See scatter plots of $\beta$ vs. density in Fig. 2 and local Mach number vs. density in Fig. 3). In addition to the "classical" parallel transport there is clear evidence of "anomalous" transport perpendicular to the flux surfaces. 
We now examine this phenomenon in more detail and compare and contrast between HFS and LFS pellet launches. Fig. 4 shows poloidal slices at the mean toroidal pellet location for the HFS and LFS cases. At time $t=2$ we observe the ablated mass in a cloud around the mean pellet position. At later times $(t=20,60)$, the pellet ablated mass has a significant outward radial displacement compared to the mean pellet location.

The LFS case shows a dramatic turning around of the mass due to the zero mass flux boundary conditions (Fig. 4-b3) It is conjectured that an outflow boundary condition would lead to a substantial loss of the ablated mass and thus poor fueling efficiency in the LFS case. The observed outward displacement implies that HFS launches are more favorable for refueling tokamaks as opposed to the LFS launches, consistent with observed behavior in experiments $[12,1]$. We may reconcile this seemingly "anomalous" transport by appealing to the model by Parks [13] which notes that magnetic curvature and $\nabla B$-induced charged particle drifts cause a local separation of charges in the pellet cloud. This leads to an axially-oriented electric field, and so the $E \times B$ drift is radially outward in both the LFS and the HFS case.

It is instructive to examine the flow pattern of the perpendicular drift velocity $v_{\perp}=E \times B /|B|^{2}$. The radial component of $v_{\perp}$ is the dominant one and is shown in Fig. 5 in a poloidal slice. For the HFS case, in Fig. 5(a), there is a dominant outward radial $v_{\perp}$ carrying the bulk of the pellet mass outward. This is flanked on either side in the axial direction by inward radial motion resulting in a nearly incompressible flow pattern. So the simple picture of only outward radial $v_{\perp}$ drift is augmented by this somewhat smaller turning around of the mass which leads to the mushroom-shaped structure in the poloidal plane.

For the LFS case too, the outward radial $E \times B$ drift grows with time and is clearly seen in Fig. 5(b). The perpendicular transport of the ablated mass brings into question some of the assumptions made in the earlier section. In calculation of the ablation rate, we assumed that the ablated mass is heated instantaneously to the flux surface temperature. However, the motion of the ablated mass radially outwards in the HFS case means that the temperature the pellet encounters will actually be smaller than that assumed. Furthermore, the energy sink term in the equations, which are based on a one-dimensional parallel transport model will need to be modified.

\section{Conclusion and Future Work}

In this paper, we presented a numerical method which is based on an unsplit upwinding method coupled with the eight-wave formulation. A MACprojection scheme is implemented to enforce the solenoidal property of the 
magnetic field. This projection requires the solution of a Poisson equation which is solved using a multi-grid technique. It was observed that the convergence of the Poisson solver was sensitive to the block-size in the AMR mesh-hierarchy. A pellet injection model was implemented as a source term in the density equations and corresponding energy sources and sinks in the energy equation. AMR simulations of the pellet injection process were carried out for the inside and outside launch cases. Preliminary studies indicate that AMR provides a speed-up exceeding two orders of magnitude over corresponding uniform mesh simulations essential to accurately resolve the physical processes involved in pellet injection. AMR is an effective way of achieving computational efficiency in detailed and resolved simulations of the pellet injection process. It was observed that the pellet ablated mass is dominantly transported along magnetic field lines but that a $E \times B$ drift causes a significant outward radial motion of the pellet cloud in both the LFS and HFS cases. A high resolution numerical simulation is a viable method of computing the relative importance of these two competing phenomena for redistributing the pellet mass.

The results presented in this paper did not include resistive terms which will be included in future work. We also plan to develop models which better account for the rapid electron heating and corresponding cooling terms in the energy equation, taking into account the perpendicular transport of the ablated mass. Finally, we plan to undertake resolved simulations of pellet injection with more realistic physical parameters, and to investigate other launch locations in addition to the HFS and LFS pellet injections.

\section{Acknowledgment}

The authors benefitted from useful discussions with Drs. W. Park, P. Parks, H. Strauss, and G. Schmidt. This work was supported by USDOE Contract no. DE-AC020-76-CH03073. This research used resources of the National Energy Research Scientific Computing Center, which is supported by the Office of Science of the U.S. Department of Energy under Contract No. DE-AC03$76 \mathrm{SF} 00098$.

\section{References}

[1] L. Baylor et al., Phys. Plasmas, vol 32, pp:1878, 2000.

[2] L. Baylor et al., J. Nucl. Matter, vol 313, pp:530, 2003.

[3] H. R. Strauss and W. Park, Phys. Plasmas, vol 5, pp:2676, 1998. 
[4] P. B. Parks and R. J. Turnbull, Phys. Fluids, vol. 21, pp:1735, 1978.

[5] A. K. Macaulay. Nuclear Fusion, vol. 34, pp:43, 1994.

[6] S. K. Godunov. Numer. Methods Mech. Contin. Media, vol 1, pp:26, 1972.

[7] K. G. Powell et al. J. Comput. Phys., vol. 154, pp:284-309, 1999.

[8] B. V. Kuteev. Nuclear Fusion, vol. 35, pp:431, 1995.

[9] P. Colella, J. Comput. Phys., vol 87, pp: 171-200, 1990.

[10] R. Crockett et al. J. Comput. Phys. (submitted), 2003.

[11] http://seesar.lbl.gov/ANAG/chombo.

[12] P. T. Lang et al., Phys. Rev. Lett., vol 79, pp:1487, 1997.

[13] P. B. Parks and W. D. Sessions and L. R. Baylor, Phys. Plasmas, vol. 7, pp:1968, 2000. pp:431, 1995.

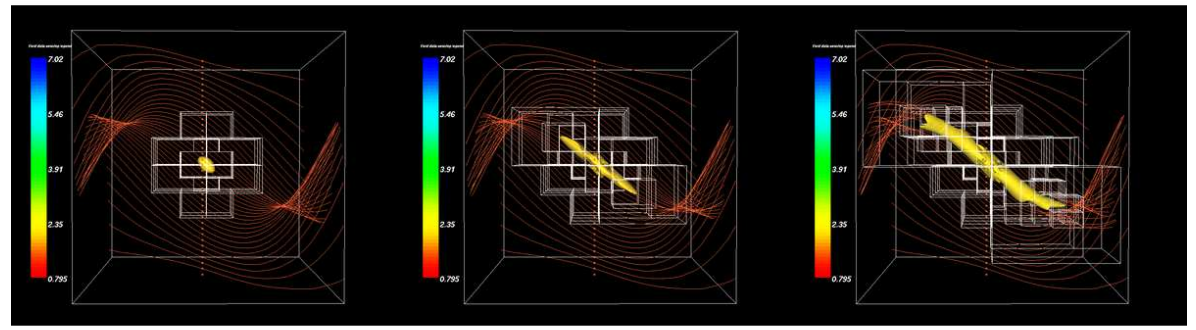

Fig. 1. Density isosurface $(\rho=2)$ for a HFS pellet launch. (a) $t=2$ (b) $t=20$ (c) $t=60$ viewed radially inwards. The magnetic field lines are shown in red. The box outlines depict the meshes. Time is normalized by the Alfvèn wave transit time. (Note that altough the domain is a torus the visualizations are presented in a cube)

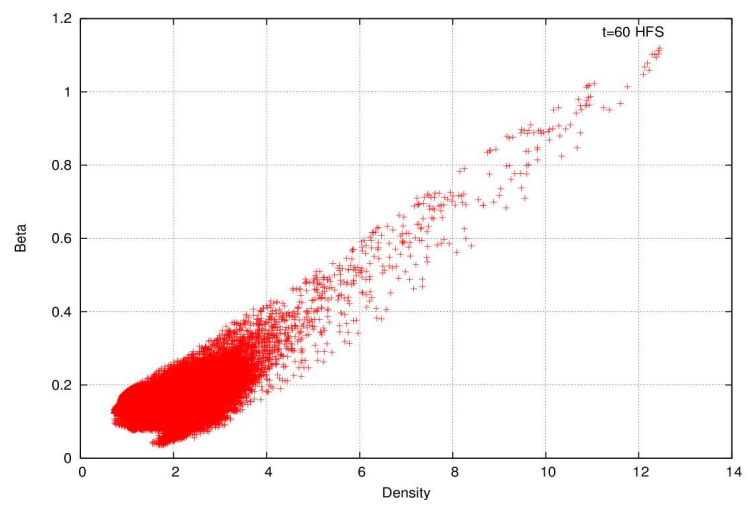

Fig. 2. Scatter plot of $\beta$ vs. $\rho$ at $t=60$ for the HFS launch. High $\beta$ is strongly correlated with high density. 


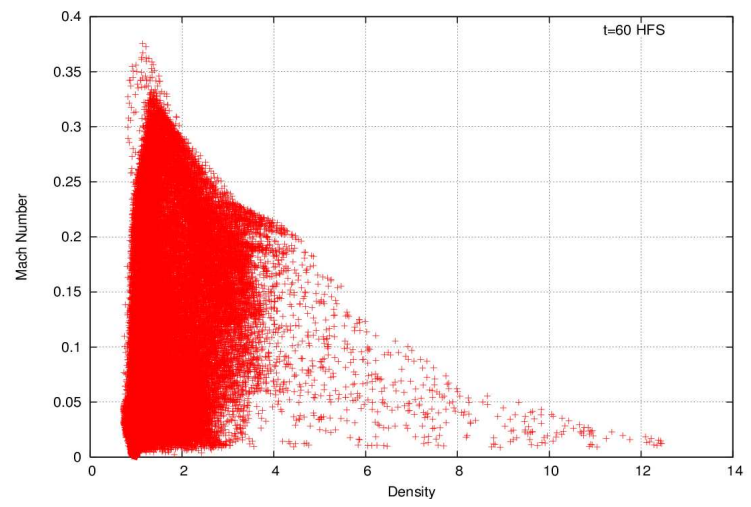

Fig. 3. Scatter plot of local Mach number vs. $\rho$ at $t=60$ for the HFS launch.
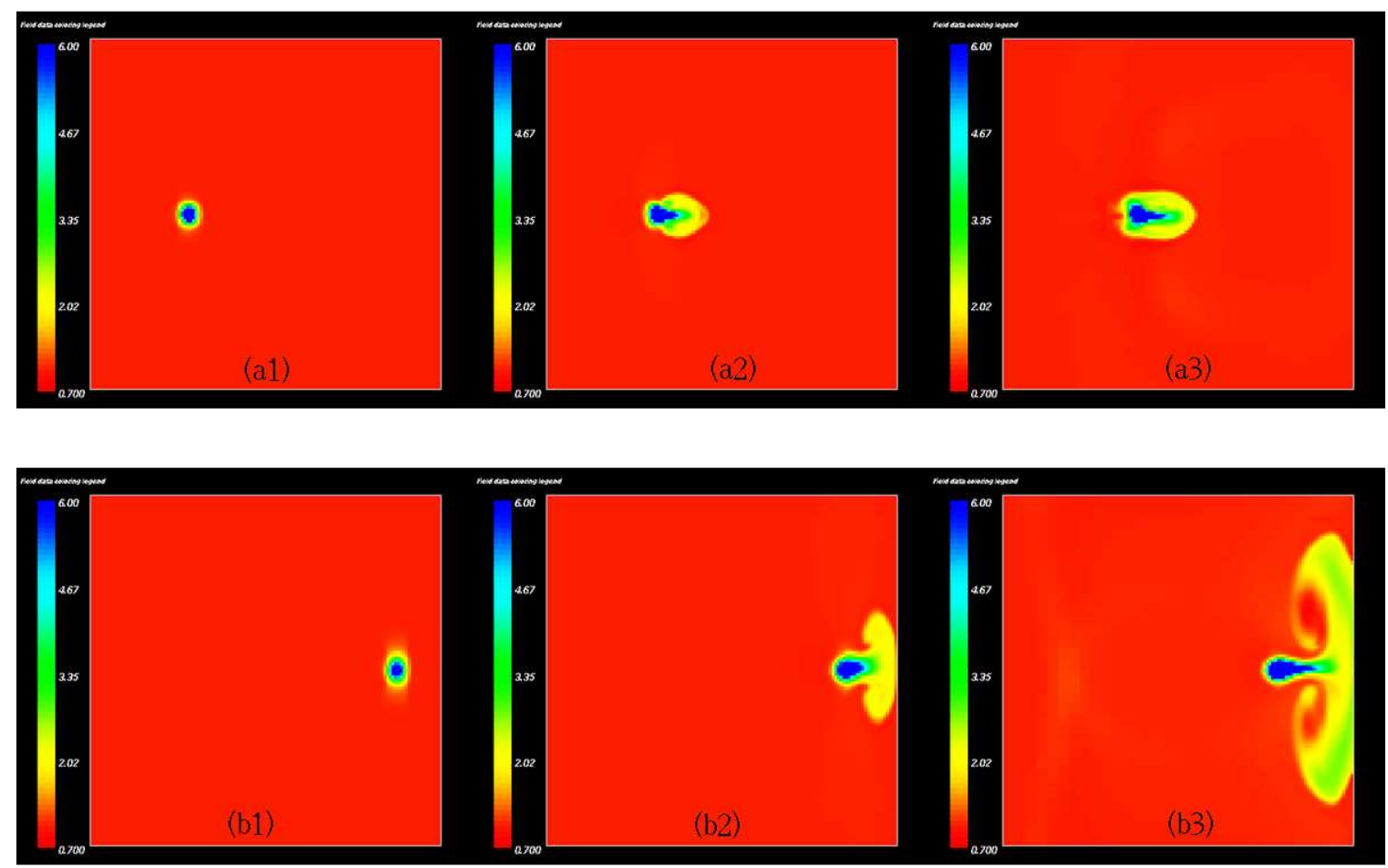

Fig. 4. Density field in a poloidal cross-section. (a1) HFS $t=2$, (a2) HFS $t=20$, (a3) HFS $t=60$, (b1) LFS $t=2$, and (b2) LFS $t=20$, and (b3) LFS $t=60$. 

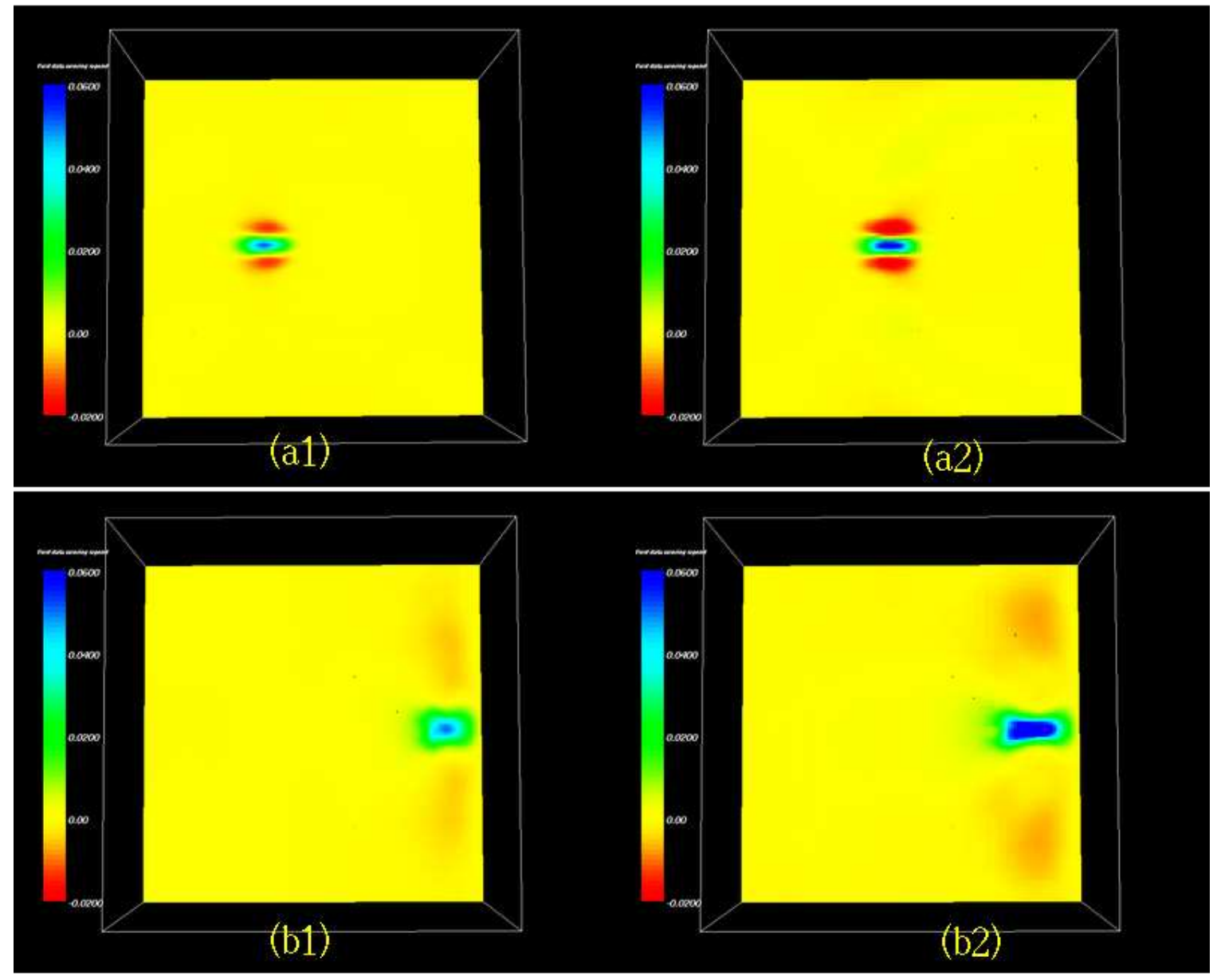

Fig. 5. Perpendicular drift velocity $v_{\perp}$ in a Poloidal cross-section. (a1) HFS $t=20$, (a2) HFS $t=60$, (b1) LFS $t=20$, and (b2) LFS $t=60$. 


\section{External Distribution}

Plasma Research Laboratory, Australian National University, Australia

Professor I.R. Jones, Flinders University, Australia

Professor João Canalle, Instituto de Fisica DEQ/IF - UERJ, Brazil

Mr. Gerson O. Ludwig, Instituto Nacional de Pesquisas, Brazil

Dr. P.H. Sakanaka, Instituto Fisica, Brazil

The Librarian, Culham Laboratory, England

Mrs. S.A. Hutchinson, JET Library, England

Professor M.N. Bussac, Ecole Polytechnique, France

Librarian, Max-Planck-Institut für Plasmaphysik, Germany

Jolan Moldvai, Reports Library, Hungarian Academy of Sciences, Central Research Institute for Physics, Hungary

Dr. P. Kaw, Institute for Plasma Research, India

Ms. P.J. Pathak, Librarian, Institute for Plasma Research, India

Ms. Clelia De Palo, Associazione EURATOM-ENEA, Italy

Dr. G. Grosso, Instituto di Fisica del Plasma, Italy

Librarian, Naka Fusion Research Establishment, JAERI, Japan

Library, Laboratory for Complex Energy Processes, Institute for Advanced Study, Kyoto University, Japan

Research Information Center, National Institute for Fusion Science, Japan

Dr. O. Mitarai, Kyushu Tokai University, Japan

Dr. Jiangang Li, Institute of Plasma Physics, Chinese Academy of Sciences, People's Republic of China

Professor Yuping Huo, School of Physical Science and Technology, People's Republic of China

Library, Academia Sinica, Institute of Plasma Physics, People's Republic of China

Librarian, Institute of Physics, Chinese Academy of Sciences, People's Republic of China

Dr. S. Mirnov, TRINITI, Troitsk, Russian Federation, Russia

Dr. V.S. Strelkov, Kurchatov Institute, Russian Federation, Russia

Professor Peter Lukac, Katedra Fyziky Plazmy MFF UK, Mlynska dolina F-2, Komenskeho Univerzita, SK-842 15 Bratislava, Slovakia

Dr. G.S. Lee, Korea Basic Science Institute, South Korea

Institute for Plasma Research, University of Maryland, USA

Librarian, Fusion Energy Division, Oak Ridge National Laboratory, USA

Librarian, Institute of Fusion Studies, University of Texas, USA

Librarian, Magnetic Fusion Program, Lawrence Livermore National Laboratory, USA

Library, General Atomics, USA

Plasma Physics Group, Fusion Energy Research Program, University of California at San Diego, USA

Plasma Physics Library, Columbia University, USA

Alkesh Punjabi, Center for Fusion Research and Training, Hampton University, USA

Dr. W.M. Stacey, Fusion Research Center, Georgia Institute of Technology, USA

Dr. John Willis, U.S. Department of Energy, Office of Fusion Energy Sciences, USA

Mr. Paul H. Wright, Indianapolis, Indiana, USA 
The Princeton Plasma Physics Laboratory is operated by Princeton University under contract with the U.S. Department of Energy.

\author{
Information Services \\ Princeton Plasma Physics Laboratory \\ P.O. Box 451 \\ Princeton, NJ 08543
}

Phone: 609-243-2750

Fax: 609-243-2751

e-mail: pppl_info@pppl.gov

Internet Address: http://www.pppl.gov 